\title{
Decreased nocturnal heart rate variability and potentially related brain regions in arteriosclerotic cerebral small vessel disease
}

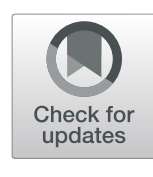

Miaoyi Zhang ${ }^{1,2}$, Huan Yu², Weijun Tang ${ }^{2}$, Ding Ding ${ }^{2}$, Jie Tang ${ }^{2}$, Na Liu², Yang Xue ${ }^{2}$, Xue Ren², Langfeng Shi ${ }^{2}$ and Jianhui $\mathrm{Fu}^{1,2^{*}}$

\begin{abstract}
Background: To assess heart rate variability (HRV) among patients with arteriosclerotic cerebral small vessel disease (CSVD) by comparing with control subjects, and to determine whether HRV parameters were related to structural alterations in brain regions involved in autonomic regulation among CSVD patients.

Methods: We consecutively recruited subjects aged between 50 and 80 years who visited the Stroke Prevention Clinic of our hospital and have completed brain magnetic resonance imaging examination from September 1, 2018 to August 31, 2019. Polysomnography and synchronous analyses of HRV were then performed in all participants. Multivariable binary logistic regression was used to identify the relationship between HRV parameters and CSVD. Participants were invited to further undergo three-dimensional brain volume scan, and the voxel based morphometry (VBM) analysis was used to identify gray matter atrophy.
\end{abstract}

Results: Among 109 participants enrolled in this study, 63 were assigned to the arteriosclerotic CSVD group and 46 to the control group. Lower standard deviation of normal-to-normal intervals (SDNN, OR $=0.943,95 \% \mathrm{Cl} 0.903$ to $0.985, P=0.009$ ) and higher ratio of low to high frequency power (LF/HF, OR=4.372, 95\% Cl 1.033 to $18.508, P=$ 0.045) during the sleep period were associated with CSVD, independent of traditional cerebrovascular risk factors and sleep disordered breathing. A number of 24 CSVD patients and 21 controls further underwent threedimensional brain volume scan and VBM analysis. Based on VBM results, SDNN during the awake time $(\beta=0.544$, $95 \% \mathrm{Cl} 0.211$ to $0.877, P=0.001)$ and the sleep period $(\beta=0.532,95 \% \mathrm{Cl} 0.202$ to $0.862, P=0.001)$ were both positively related with gray matter volume within the right inferior frontal gyrus only among CSVD patients.

Conclusions: Decreased nocturnal HRV is associated with arteriosclerotic CSVD independent of traditional cerebrovascular risk factors and sleep disordered breathing. The structural atrophy of some brain regions associated with cardiac autonomic regulation sheds light on the potential relationship.

Trial registration: Trial registration number: ChiCTR1800017902. Date of registration: 20 Aug 2018.

Keywords: Arteriosclerotic cerebral small vessel disease, Heart rate variability, Nocturnal, Gray matter atrophy

\footnotetext{
* Correspondence: jianhuifu@126.com

'Department of Neurology, North Huashan Hospital, Fudan University, No.108 Lu Xiang Road, Shanghai 201900, China

2Department of Neurology, Huashan Hospital, Fudan University, No.12

Wulumuqi Zhong Road, Shanghai 200040, China
}

\section{$\triangle B M C$}

(c) The Author(s). 2021 Open Access This article is licensed under a Creative Commons Attribution 4.0 International License, which permits use, sharing, adaptation, distribution and reproduction in any medium or format, as long as you give appropriate credit to the original author(s) and the source, provide a link to the Creative Commons licence, and indicate if changes were made. The images or other third party material in this article are included in the article's Creative Commons licence, unless indicated otherwise in a credit line to the material. If material is not included in the article's Creative Commons licence and your intended use is not permitted by statutory regulation or exceeds the permitted use, you will need to obtain permission directly from the copyright holder. To view a copy of this licence, visit http://creativecommons.org/licenses/by/4.0/. The Creative Commons Public Domain Dedication waiver (http://creativecommons.org/publicdomain/zero/1.0/) applies to the data made available in this article, unless otherwise stated in a credit line to the data. 


\section{Introduction}

Cerebral small vessel disease (CSVD), a major contributor to stroke and cognitive impairment, is a group of diseases that pathologically affect the small arteries, arterioles, capillaries and venules of the brain $[1,2]$. The hallmark neuro-imaging markers of CSVD include recent small subcortical infarcts, lacunes of presumed vascular origin, white matter hyperintensities (WMH) of presumed vascular origin, enlarged perivascular spaces (EPVSs), cerebral microbleeds (CMBs), and brain atrophy [3]. Arteriosclerotic CSVD is one of the most prevalent forms and is strongly associated with aging and hypertension [2]. To date, the pathogenesis of arteriosclerotic CSVD has not been completely elucidated.

Heart rate variability (HRV) is considered to reflect the activity of the autonomic nervous system (ANS) [4]. The relationship between HRV and cardiovascular mortality has been reported in previous epidemiological studies [5, 6]. Recently, accumulating evidence has reported an association between HRV, particularly at nighttime, and the development and presence of subclinical arteriosclerotic CSVD [7, 8]. However, the conclusions on this topic remain controversial. In addition, sleep disorders, particularly sleep disordered breathing (SDB), were not considered as confounding factors in those studies, which in fact are increasingly recognized as risk factors for arteriosclerotic CSVD $[9,10]$ and affect HRV $[11,12]$.

The set of brain regions involved in autonomic modulation has been referred to as the central autonomic network (CAN), including the insula, cingulate cortex, medial prefrontal cortex, amygdala, and thalamus [13]. Changes in the morphology of the autonomic circuits have been reported to contribute to a sympathetic-parasympathetic imbalance [14]. Recently, brain atrophy has been considered one of the neuroimaging features of CSVD [3] and is thought to partially mediate the effects of vascular lesions on cognition [15]. Nevertheless, studies on the association between CSVD-related brain atrophy and autonomic dysfunction are scarce.

For these reasons, a plausible hypothesis is that decreased HRV, which is associated with sympathetic overactivity, may be present in patients with arteriosclerotic CSVD, and some structural alterations in cortical regions related to the CAN may play a role in the association. Thus, the current study aimed to assess HRV parameters among patients with arteriosclerotic CSVD and control subjects. The group comparisons were then determined after adjusting for traditional cerebrovascular risk factors and sleep apnea. We further sought to investigate whether the parameters were related to structural alterations in brain regions involved in autonomic regulation.

\section{Material and methods}

\section{Ethics statement}

This study conformed with the World Medical Association Declaration of Helsinki and was approved by the Huashan Hospital Research Ethics Committee (ProjectID: KY2018-224). All patients or their relatives provided written informed consent.

\section{Subjects}

We consecutively recruited subjects aged between 50 and 80 years who visited the Stroke Prevention Clinic of our hospital and have completed brain magnetic resonance imaging (MRI) examination from September 1, 2018 to August 31, 2019. The MRI sequences included T1-weighted, T2-weighted, fluid-attenuated inversion recovery, and susceptibility weighted imaging, using a 3Tesla scanner (Siemens Magneton Verio $3 \mathrm{~T}$ ).

The inclusion criteria for patients diagnosed with arteriosclerotic CSVD included 1) baseline MRI scan mainly showing moderate to severe WMH of presumed vascular origin (Fazekas score of 2-3), with or without the presence of other MRI features of CSVD (lacune of presumed vascular origin, CMBs, EPVSs and brain atrophy) [3]; 2) one or more characteristic clinical manifestations of CSVD (including cognitive, motor or mood disturbances) or no evident symptoms; and (3) consent to participate in the study. The exclusion criteria were: 1) cortical infarct or large subcortical infarct $(>2 \mathrm{~cm})$ on conventional MRI; 2) previous ischemic stroke which was less than 6 months after onset; 3) carotid artery stenosis $\geq 50 \%$ [16]; 4) non-arteriosclerotic CSVD, such as inherited CSVD or probable cerebral amyloid angiopathy (CAA); 5) any other cause of white matter disease; 6) major psychiatric disorders; 7) the presence of any ANS disorders or clinically relevant arrhythmia; and 8) a systemic or terminal illness that could not complete examinations.

Individuals in the control group were those who visited the Stroke Prevention Clinic with dizziness or headache as chief complaints, but without characteristic neuroimaging markers of CSVD on baseline MRI, except for mild WMH of presumed vascular origin (Fazekas score of $0-1$ ) and age-matched brain atrophy. Other inclusion criteria included: no history of definite cerebrovascular disease, Parkinson's disease, cognitive impairment or psychiatric disorders; and consent to participate in the study. Enrollment exclusions were: 1) a systemic or terminal illness that could not complete examinations; and 2) the presence of any ANS disorders or clinically relevant arrhythmia.

\section{Traditional cerebrovascular risk factors}

Cerebrovascular risk factors were ascertained through laboratory examinations and interviews conducted by 
experienced physicians. Hypertension was defined as systolic blood pressure $>140 \mathrm{mmHg}$ or diastolic blood pressure $\geq 90 \mathrm{mmHg}$, or the use of antihypertensive drugs [17]. Diabetes mellitus (DM) was defined as glycated hemoglobin level $\geq 6.5 \%$, fasting glucose level $\geq 126$ $\mathrm{mg} / \mathrm{dL}$, 2-h glucose level $\geq 200 \mathrm{mg} / \mathrm{dL}$, or the current use of insulin or hypoglycemic agents [18]. Hyperlipidemia was defined as total serum cholesterol level $\geq 5.9$ $\mathrm{mmol} / \mathrm{L}$, total triglyceride level $\geq 1.8 \mathrm{mmol} / \mathrm{L}$, or the use of lipid-lowering medications. Previous stroke was defined as the presentation of sudden focal neurological deficits with consistent radiological findings occurred more than 6 months before enrollment. The smoking history (including ex- and current smoker) and body mass index (BMI) were also recorded.

\section{Polysomnography (PSG)}

Eligible subjects in two groups were then referred to the Sleep Center to subsequently complete the PSG examination. Compumedics Profusion PSG V4.5 (Shanghai, China) was used to monitor sleep parameters for a whole night of sleep, and the permission for its application was obtained. Pittsburgh Sleep Quality Index (PSQI) was applied to measure the subjective sleep quality during the last month. All subjects were instructed not to use sleep medications, anxiolytics, or antidepressants for at least 2 weeks preceding the examination and not to consume caffeinated beverages, alcohol or strong tea at the afternoon preceding the recording. The monitoring time ranged from approximately 22:00 pm to $6: 30 \mathrm{am}$ the next day and was adjusted according to the individual's habitual bedtime. Sleep stages, including non-rapid eye movement (NREM) sleep (stage N1, stage N2, stage N3) and rapid eye movement (REM) sleep (stage R), were manually scored by an experienced polysomnographic technologist according to criteria from American Academy of Sleep Medicine [19] (AASM, version 2.4), who was blinded to the medical history of all participants.

Sleep apnea was defined as $a \geq 90 \%$ reduction in airflow from baseline lasting more than $10 \mathrm{~s}$ (s). Sleep hypopnea was defined as a $\geq 30 \%$ reduction in airflow from baseline lasting at least $10 \mathrm{~s}$ that was associated with either an oxygen desaturation of $>3 \%$ or an arousal. The apnea-hypopnea index (AHI) was defined as the total number of sleep apnea and hypopnea events per hour during the whole night of sleep. AHI in the NREM sleep and REM sleep were also analyzed. Hypoxiarelated parameters were also recorded, including the average oxygen saturation $\left(\mathrm{SaO}_{2}\right)$, minimum $\mathrm{SaO}_{2}$, average oxygen desaturation, oxygen desaturation index (ODI), time with $\mathrm{SaO}_{2}<90 \%$ (ST90\%), and percentage of cumulative time with $\mathrm{SaO}_{2}<90 \%$ (CT90\%). The ODI referred to the total number of $3 \%$ or greater oxygen desaturation events per hour during sleep. The periodic limb movements index (PLMI) was also calculated as the total number of limb movement events per hour during sleep.

\section{HRV analyses}

Electrocardiogram (ECG) data were obtained simultaneously during awake and sleep periods with PSG between the onset and the end of recording. The ECG Add-on for Profusion PSG 4 was applied to analyze the HRV, with a focus on time and frequency domains. Three time-domain measures of HRV were evaluated $[11,20,21]$ : the standard deviation of normal-to-normal intervals $(\mathrm{SDNN})$ which characterizes overall HRV, the root mean square of successive differences in RR intervals (RMSSD) and the percentage of normal R-R intervals that differ by $50 \mathrm{~ms}$ (PNN50) which are thought to reflect parasympathetic nervous system activity. As a frequency domain measure, the ratio of low to high frequency power (LF/HF) was calculated to estimate the balance between sympathetic and parasympathetic nervous system activity [20, 21]. HRV parameters during awake and sleep periods defined by PSG were recorded and analyzed separately.

\section{MRI data acquisition and image processing}

Based on the MRI results at baseline, all hallmark imaging markers were defined according to the STandards for ReportIng Vascular changes on nEuroimaging (STRIVE) guidelines [3]. The total CSVD scores were calculated according to previous descriptions [22], with a maximum score of four points.

Enrolled patients were invited to further undergo imaging with a three-dimensional brain volume (3DBRAVO) sequence within 1 week (acquisition parameters: $\mathrm{TR}=8.8 \mathrm{~ms}, \mathrm{TE}=1.0 \mathrm{~ms}$, flip angle $=15^{\circ}$, slice thickness $=1 \mathrm{~mm} \quad$ iso-voxel, $\quad$ FOV $=320 \mathrm{~mm} \times 320 \mathrm{~mm}$, matrix $=320 \times 320$, voxel $=1 \mathrm{~mm} \times 1 \mathrm{~mm} \times 1 \mathrm{~mm}$ ). Voxelbased morphometry (VBM) was implemented using SPM12 on the MATLAB 2016b workstation. The origin of each participant's image was adjusted to the anterior commissure. Images were segmented into gray matter, white matter, and cerebrospinal fluid using a classical unified segmentation approach with SPM12. Diffeomorphic Anatomical Registration Through Exponentiated Lie algebra (DARTEL) algorithm was used to establish a group-level template for the segmented gray matter images. Then the gray matter images of all subjects were matched to the standard human brain template space of the Montreal Neurological Institute (MNI) using the constructed template. The 3D gray matter images were modulated after high-dimensional registration and then spatially smoothed using a Gaussian filter with a full width at half maximum value of 12 
$\mathrm{mm}$ to reduce the image noise. Finally, the brain of each subject was divided according to the AnatomyToolbox atlas. The gray matter volume of each brain region was measured quantitatively by calculating the sum of all the voxels classified as gray matter, with voxel size of $1 \mathrm{~mm}$ isotropic. Parametric statistical tests were performed for group comparisons of the smoothed gray matter map after including age, sex and AHI as covariates. Differences were considered significant with a family-wise error cluster corrected probability of $p<0.0001$.

\section{Statistical analyses}

The statistical analyses were performed using SPSS 26.0 and STATA software. The categorical variables were presented as counts and percentages. The continuous variables were presented as medians (interquartile ranges [IQR]). Group comparisons were performed using the chi-squared test for categorical data, Mann-Whitney U test for non-normally distributed continuous data, and $\mathrm{t}$ test for normally distributed continuous data. The odds ratios (OR) and 95\% confidence intervals (CI) for the relationship between HRV parameters and arteriosclerotic CSVD were assessed through multivariate binary logistic regression models. Generalized linear models (GLMs) were applied to analyze the relationships between HRV parameters and gray matter volume in the two groups. Due to the small sample size, variables with a $P$ value $<$ 0.05 in the group comparisons were preferentially entered into the models.

\section{Results}

\section{Patient characteristics}

A total of 115 eligible subjects were selected in the current study. Sixty-five patients were diagnosed with arteriosclerotic CSVD, two of whom were excluded because they failed to complete PSG tests. Fifty subjects were assigned to the control group, one of whom was excluded because of severe sleep apnea-hypopnea syndrome (SAHS) with objective cognitive dysfunction and three were excluded because of incomplete PSG records. Thus, 63 patients with arteriosclerotic CSVD and 46 controls were included in the final analysis. The baseline characteristics are summarized in Table 1.

Sixty-five point one percent $(41 / 63)$ of patients with arteriosclerotic CSVD (median age 68.0 years) were men, while subjects in the control group (median age 69.0 years) comprised of $32.6 \%(15 / 46)$ men. Difference in the sex composition of the two groups was observed $(P=$ 0.001). Significant differences were also noted in the incidence of hypertension, symptomatic stroke and hyperlipidemia in patients with CSVD, as well as the use of anti-hypertension drugs and BMI. Regarding sleep parameters, patients with CSVD showed shorter duration of REM sleep $(P=0.014)$ compared with the control group and showed an overall increase in the AHI, including in the whole night of sleep $(P<0.001)$, NREM sleep $(P=0.004)$ and REM sleep $(P<0.001)$. The group differences in all hypoxia-related parameters also reached statistical significance, including the average $\mathrm{SaO}_{2}$, minimum $\mathrm{SaO}_{2}$, average oxygen desaturation, ODI, ST $90 \%$ and CT90\%. In addition, age, the incidence of DM, smoking history, PSQI and PLMI were not significantly different between the two groups.

\section{HRV analyses}

A nonparametric test revealed significant differences in the average heart rate $(P=0.003)$, SDNN $(P=0.001)$ and LF/HF $(P=0.006)$ during the sleep period between the two groups, but significant differences were not observed in any of the other parameters (Table 1). The results of binary logistic regression analyses between HRV parameters and arteriosclerotic CSVD are presented in Table 2. After adjusting for sex, hypertension, previous stroke, hyperlipidemia, and BMI (Model 1), patients with arteriosclerotic CSVD had higher LF/HF ratios during both awake $(\mathrm{OR}=2.776,95 \% \mathrm{CI} 1.093$ to $7.055, P=$ $0.032)$ and sleep periods $(\mathrm{OR}=5.853,95 \%$ CI 1.626 to 21.063, $P=0.007$ ) than the control group. When simultaneously entering multiple variables in Model 1 , AHI, ODI and the interaction of the latter two variables (Model 2), significant differences were observed in SDNN (OR $=0.943,95 \%$ CI 0.903 to $0.985, P=0.009)$ and LF/HF during the sleep period $(\mathrm{OR}=4.372,95 \% \mathrm{CI}$ 1.033 to $18.508, P=0.045$ ) between the two groups.

\section{Gray matter volume and relationships with autonomic parameters}

Complete neuroimaging data were available for only 45 participants: 24 in the arteriosclerotic CSVD group and 21 in the control group. Participant characteristics are presented in Table 3 . The median age was 63.5 years in the CSVD group, and 69.0 years in the control $\operatorname{group}(P=$ $0.014)$. The incidences of hypertension $(P<0.001)$, previous symptomatic stroke $(P<0.001)$, hyperlipidemia $(P<$ $0.001)$ differed significantly between the two groups. Patients with CSVD showed a higher AHI $(P=0.004)$. Statistical significance existed in SDNN during the sleep period $(P=0.009)$, PNN50 during the awake $(P=0.024)$ and sleep periods $(P=0.006)$ between patients with CSVD and controls. No statistical differences were noted between the two groups in the sex composition, the incidence of DM, smoking history, PSQI, BMI, PLMI during sleep period and other HRV parameters. As for radiologic features, all participants in the control group showed no signs of lacunar infarcts, $\mathrm{CMB}$ and moderate to extensive EPVSs in basal ganglia. Among patients with arteriosclerotic CSVD, extensive $\mathrm{WMH}(91.7 \%)$ and the presence of $\mathrm{CMB}(91.7 \%)$ were the most prevalent, 
Table 1 Baseline characteristics of CSVD and control group

\begin{tabular}{|c|c|c|c|}
\hline & CSVD group $(n=63)$ & Control group $(n=46)$ & $P$ \\
\hline Sex (male / famale) & $41 / 22$ & $15 / 31$ & $0.001^{*}$ \\
\hline Age, years, median (IQR) & $68.0(62.0,73.0)$ & $69.0(65.0,71.0)$ & 0.455 \\
\hline Hypertension, $n(\%)$ & $52(82.5)$ & $8(17.4)$ & $<0.001^{*}$ \\
\hline $\mathrm{DM}, \mathrm{n}(\%)$ & $11(17.5)$ & $4(8.7)$ & 0.190 \\
\hline History of symptomatic stroke, n(\%) & $28(44.4)$ & $0(0.0)$ & $<0.001^{*}$ \\
\hline Hyperlipidemia, n(\%) & $46(73.0)$ & $9(19.6)$ & $<0.001^{*}$ \\
\hline Smoking history, n(\%) & $15(23.8)$ & $5(10.9)$ & 0.085 \\
\hline \multicolumn{4}{|l|}{ Anti-hypertension drugs, n(\%) } \\
\hline ACEI/ARB & $33(52.4)$ & $5(10.9)$ & $<0.001^{*}$ \\
\hline B-receptor blocker & $11(17.5)$ & $2(4.3)$ & $0.037^{*}$ \\
\hline a-receptor blocker & $4(6.3)$ & $0(0.0)$ & 0.220 \\
\hline aß-receptor blocker & $1(1.6)$ & $0(0.0)$ & 1.000 \\
\hline$C C B$ & $36(57.1)$ & $6(13.0)$ & $<0.001^{*}$ \\
\hline diuredics & $5(7.9)$ & $1(2.2)$ & 0.380 \\
\hline $\mathrm{BMI}, \mathrm{kg} / \mathrm{m}^{2}$, median (IQR) & $25.1(22.2,27.7)$ & $22.3(20.4,25.4)$ & $0.010^{*}$ \\
\hline PSQI, median (IQR) & $8.0(4.0,10.0)$ & $7.0(4.8,10.0)$ & 0.985 \\
\hline \multicolumn{4}{|l|}{ Sleep Stage, \%, median (IQR) } \\
\hline Stage N1 & $8.3(5.7,14.3)$ & $7.2(5.2,10.3)$ & 0.064 \\
\hline Stage N2 & $50.3(44.4,58.8)$ & $51.3(45.2,57.5)$ & 0.895 \\
\hline Stage N3 & $19.8(11.0,28.4)$ & $20.0(15.8,26.9)$ & 0.854 \\
\hline Stage R & $16.7(12.1,21.5)$ & $19.0(16.3,22.2)$ & $0.014^{*}$ \\
\hline AHI, times/hour, median (IQR) & $22.4(12.6,31.4)$ & $10.8(6.4,18.2)$ & $<0.001^{*}$ \\
\hline AHI in NREM sleep & $18.8(9.4,30.3)$ & $8.0(3.6,15.6)$ & $0.004^{*}$ \\
\hline AHI in REM sleep & $36.6(18.3,51.9)$ & $23.3(14.7,35.0)$ & $<0.001^{*}$ \\
\hline Average $\mathrm{SaO}_{2}, \%$, median (IQR) & $94.0(92.0,95.0)$ & $95.0(94.0,96.0)$ & $0.005^{*}$ \\
\hline Minimum $\mathrm{SaO}_{2}, \%$, median (IQR) & $85.0(81.0,89.0)$ & $89.0(84.3,90.8)$ & $0.013^{*}$ \\
\hline Average oxygen desaturation, \%, median (IQR) & $4.0(4.0,6.0)$ & $4.0(4.0,4.8)$ & $0.011^{*}$ \\
\hline ODI, times/hour, median (IQR) & $14.6(7.4,25.8)$ & $5.7(2.4,12.4)$ & $<0.001^{*}$ \\
\hline ST90\%, min, median (IQR) & $3.1(0.3,17.6)$ & $0.8(0.0,7.3)$ & $0.013^{*}$ \\
\hline СT90\%, \%, median (IQR) & $1.3(0.1,7.4)$ & $0.2(0.0,1.8)$ & $0.008^{*}$ \\
\hline PLMI during sleep period, times/hour, median (IQR) & $1.7(0.0,10.1)$ & $1.7(0.0,10.9)$ & 0.933 \\
\hline Average HR during the sleep period, times/min, median (IQR) & $67.0(60.0,74.0)$ & $62.0(57.8,67.3)$ & $0.003^{*}$ \\
\hline \multicolumn{4}{|l|}{ HRV during awake period, median (IQR) } \\
\hline SDNN & $68.7(54.7,89.4)$ & $73.5(62.5,83.3)$ & 0.317 \\
\hline RMSSD & $27.2(17.4,42.4)$ & $30.2(23.9,43.3)$ & 0.273 \\
\hline PNN50 & $3.2(0.9,8.9)$ & $5.5(2.8,14.2)$ & 0.059 \\
\hline LF/HF & $1.3(0.9,2.1)$ & $1.2(0.8,1.5)$ & 0.081 \\
\hline \multicolumn{4}{|l|}{ HRV during sleep period, median (IQR) } \\
\hline SDNN & $53.6(44.1,71.9)$ & $70.2(60.8,77.3)$ & $0.001^{*}$ \\
\hline RMSSD & $26.9(19.2,40.7)$ & $29.6(24.1,42.9)$ & 0.304 \\
\hline PNN50 & $3.3(1.0,10.2)$ & $6.2(2.6,14.5)$ & 0.053 \\
\hline LF/HF & $1.1(0.9,2.1)$ & $1.0(0.7,1.3)$ & $0.006^{*}$ \\
\hline
\end{tabular}

Statistical significance is reported as * $(P<0.05)$

Abbreviations: CSVD cerebral small vessel disease, $I Q R$ interquartile range, $D M$ diabetes mellitus, $A C E I$ angiotensin-converting enzyme inhibitor, $A R B$ angiotensin receptor blocker, $C C B$ calcium channel blockers, $P S Q I$ Pittsburgh Sleep Quality Index, NREM non-rapid eye movement, $R E M$ rapid eye movement, $B M I$ body mass index, AHI apnea- hypopnea index, SAHS sleep apnea-hypopnea syndrome, $\mathrm{SaO}_{2}$ oxygen saturation, ODI oxygen desaturation index, ST90\% time with $\mathrm{SaO}_{2}<90 \%$, CT90\% percentage of cumulative time with $\mathrm{SaO}_{2}<90 \%$, PLMI periodic limb movements index, HR heart rate, SDNN the standard deviation of normal-to-normal intervals, RMSSD the root mean square of successive differences in RR intervals, PNN50 the percentage of normal R-R intervals that differ by 50 ms, $L F$ lowfrequency power, HF high-frequency power 
Table 2 Results of binary logistic regression analyses between HRV parameters and CSVD

\begin{tabular}{|c|c|c|c|c|}
\hline & \multicolumn{2}{|l|}{ Model $1^{\mathrm{a}}$} & \multicolumn{2}{|l|}{ Model $2^{b}$} \\
\hline & OR (95\% Cl) & $P$ & OR $(95 \% \mathrm{Cl})$ & $P$ \\
\hline Average heart rate during the sleep period, times/min & $1.047(0.975$ to 1.124$)$ & 0.207 & $1.023(0.945$ to 1.107$)$ & 0.577 \\
\hline \multicolumn{5}{|l|}{ HRV during awake period } \\
\hline SDNN & $0.994(0.970$ to 1.108$)$ & 0.624 & $0.986(0.959$ to 1.013$)$ & 0.307 \\
\hline RMSSD & $1.002(0.980$ to 1.025$)$ & 0.857 & $0.995(0.972$ to 1.019$)$ & 0.693 \\
\hline PNN50 & $0.981(0.918$ to 1.049$)$ & 0.581 & $0.975(0.911$ to 1.043$)$ & 0.462 \\
\hline $\mathrm{LF} / \mathrm{HF}$ & $2.776(1.093$ to 7.055$)$ & $0.032^{*}$ & $2.053(0.721$ to 5.845$)$ & 0.178 \\
\hline \multicolumn{5}{|l|}{ HRV during sleep period } \\
\hline SDNN & $0.970(0.938$ to 1.002$)$ & 0.069 & $0.943(0.903$ to 0.985$)$ & $0.009^{*}$ \\
\hline RMSSD & $1.003(0.982$ to 1.026$)$ & 0.758 & $0.997(0.974$ to 1.020$)$ & 0.778 \\
\hline PNN50 & $0.982(0.922$ to 1.045$)$ & 0.558 & 0.975 (0.915 to 1.039$)$ & 0.430 \\
\hline LF/HF & $5.853(1.626$ to 21.063$)$ & $0.007^{*}$ & $4.372(1.033$ to 18.508$)$ & $0.045^{*}$ \\
\hline
\end{tabular}

Statistical significance is reported as * $(P<0.05)$

Abbreviations: HRV heart rate variability, CSVD cerebral small vessel disease, $B M I$ body mass index, $A H I$ apnea- hypopnea index, SDNN the standard deviation of normal-to-normal intervals, RMSSD the root mean square of successive differences in RR intervals, PNN50 the percentage of normal R-R intervals that differ by 50 ms, $L F$ low-frequency power, $H F$ high-frequency power

${ }^{a}$ Model 1: adjusted by sex, hypertension, previous stroke, hyperlipidemia, BMI

${ }^{\mathrm{b}}$ Model 2: Model 1+ AHI, ODI, AHI $\times \mathrm{ODI}$ (the interaction item of $\mathrm{AHI}$ and $\mathrm{ODI}$ )

followed by moderate to extensive EPVSs in basal ganglia(75.0\%) and lacunar infarcts(37.5\%). 4(16.7\%) patients with arteriosclerotic CSVD had two markers, 10 (41.7\%) had three different neuroimaging markers on MRI and all MRI features were simultaneously present in the rest(41.7\%).

Using VBM, the arteriosclerotic CSVD group presented with significant gray matter atrophy in certain brain regions, including the bilateral cerebellum, right superior frontal gyrus, right inferior frontal gyrus, right thalamus, right temporal pole, right superior temporal gyrus, right lingual gyrus, right medial cingulate cortex and left anterior cingulate cortex (Table 4 and Fig. 1).GLM analyses were conducted to determine the associations between autonomic parameters and the gray matter atrophy described above in the two groups. Age, AHI, and total burden were entered into the GLMs as covariates. Among patients with arteriosclerotic CSVD, SDNN during the awake and sleep periods were both positively related with gray matter volume within the right inferior frontal gyrus (the former: $\beta=0.544,95 \% \mathrm{CI}$ 0.211 to $0.877, P=0.001$; the latter: $\beta=0.532,95 \% \mathrm{CI}$ 0.202 to $0.862, P=0.001$ ). No evident relationship was observed between all parameters and gray matter volume in the control group.

\section{Discussion}

The current study demonstrated that patients with arteriosclerotic CSVD show more prominent heart rate fluctuations than controls during the sleep period, independent of traditional cerebrovascular risk factors and $\mathrm{SDB}$, suggesting the existence of sympathetic overactivity. In addition, using quantitative neuroimaging methods, we determined that arteriosclerotic CSVD is accompanied by structural alterations in some brain regions associated with cardiac autonomic regulation.

Regarding HRV measures, SDNN represents overall variability and joint sympathetic and parasympathetic modulation of HRV, while a higher LF/HF ratio indicates a sympathetic predominance. In the current study, lower SDNN and higher LF/HF ratios were observed during sleep among patients with arteriosclerotic CSVD, even after adjusting for SDB, illustrating the potential effect of sympathetic overactivity on the presence of arteriosclerotic CSVD, particularly during the night. Similar to some other reports, the present study did not show a significant difference in HRV during the awake period between the groups $[7,8,23]$. A possible explanation for this finding is that the prominent nocturnal heart rate fluctuations may reflect sustained sympathetic activation and accordingly exert more adverse effects on cerebral white matter [6]. Furthermore, HRV during the awake period is always influenced by many factors, such as physical activity and emotion; thus, measurement bias probably existed [6].

Based on the VBM results, we observed significant reductions in the gray matter volume of certain brain regions among patients with arteriosclerotic CSVD, some of which have been reported to be involved in the central command of cardiac autonomic modulation [14, 24-26]. However, only the right inferior frontal gyrus was shown to be more significantly related to the fluctuations of heart rate during both awake and sleep periods in our study. Interestingly, our results also suggested the 
Table 3 Characteristics of subjects included in VBM analyses

\begin{tabular}{|c|c|c|c|}
\hline & CSVD group $(n=24)$ & Control group $(n=21)$ & $P$ \\
\hline Sex (male / famale) & $15 / 9$ & $8 / 13$ & 0.102 \\
\hline Age, years, median (IQR) & $63.5(60.8,69.0)$ & $69.0(68.0,70.0)$ & $0.014^{*}$ \\
\hline Hypertension, $n(\%)$ & $21(87.5)$ & $6(28.6)$ & $<0.001^{*}$ \\
\hline $\mathrm{DM}, \mathrm{n}(\%)$ & $5(20.8)$ & $2(9.5)$ & 0.527 \\
\hline History of symptomatic stroke, $\mathrm{n}(\%)$ & $11(45.8)$ & $0(0.0)$ & $<0.001^{*}$ \\
\hline Hyperlipidemia, n(\%) & $21(87.5)$ & $4(19.0)$ & $<0.001^{*}$ \\
\hline Smoking history, n(\%) & $5(20.8)$ & $1(4.8)$ & 0.253 \\
\hline Presence of lacunar infarcts, n(\%) & $9(37.5)$ & $0(0.0)$ & $0.006^{*}$ \\
\hline Extensive $\mathrm{WMH}^{\mathrm{a}}, \mathrm{n}(\%)$ & $22(91.7)$ & $0(0.0)$ & $<0.001^{*}$ \\
\hline Moderate to extensive EPVSs in basal ganglia ${ }^{\mathrm{b}}, \mathrm{n}(\%)$ & $18(75.0)$ & $0(0.0)$ & $<0.001^{*}$ \\
\hline Presence of cerebral microbleeds, $\mathrm{n}(\%)$ & $22(91.7)$ & $0(0.0)$ & $<0.001^{*}$ \\
\hline \multicolumn{4}{|l|}{ Total CSVD burden, n(\%) } \\
\hline 1 & $0(0.0)$ & $0(0.0)$ & - \\
\hline 2 & $4(16.7)$ & $0(0.0)$ & 0.151 \\
\hline 3 & $10(41.7)$ & $0(0.0)$ & $0.003^{*}$ \\
\hline 4 & $10(41.7)$ & $0(0.0)$ & $0.003^{*}$ \\
\hline PSQI, median (IQR) & $5.5(3.0,9.3)$ & $6.0(4.0,9.0)$ & 0.515 \\
\hline $\mathrm{BMI}, \mathrm{kg} / \mathrm{m}^{2}$, median (IQR) & $24.1(22.3,27.8)$ & $22.4(20.8,25.4)$ & 0.125 \\
\hline AHI, times/hour, median (IQR) & $19.4(12.1,27.8)$ & $9.5(6.6,16.6)$ & $0.004^{*}$ \\
\hline PLMI during sleep period, times/hour, median (IQR) & $1.8(0.0,16.4)$ & $0.4(0.0,12.4)$ & 0.591 \\
\hline Average HR during the sleep period, times/min, median (IQR) & $65.5(60.8,72.5)$ & $63.0(57.0,69.0)$ & 0.127 \\
\hline \multicolumn{4}{|l|}{ HRV during awake period, median (IQR) } \\
\hline SDNN & $70.7(61.2,86.5)$ & $78.5(64.0,80.8)$ & 0.585 \\
\hline RMSSD & $28.7(19.3,41.8)$ & $29.7(25.2,44.3)$ & 0.381 \\
\hline PNN50 & $3.1(0.9,9)$ & $7.5(3.8,16.6)$ & $0.024^{*}$ \\
\hline LF/HF & $1.4(1.0,2.0)$ & $1.2(0.9,1.5)$ & 0.231 \\
\hline \multicolumn{4}{|l|}{ HRV during sleep period, median (IQR) } \\
\hline SDNN & $53.9(45.7,64.0)$ & $69.6(49.4,74.2)$ & $0.009^{*}$ \\
\hline RMSSD & $26.4(19.4,41.8)$ & $29.3(24.7,43.5)$ & 0.260 \\
\hline PNN50 & $2.9(1.0,9.3)$ & 7.4(4.2, 19.1) & $0.006^{*}$ \\
\hline $\mathrm{LF} / \mathrm{HF}$ & $1.2(0.9,1.8)$ & $1.0(0.8,1.2)$ & 0.144 \\
\hline
\end{tabular}

Statistical significance is reported as * $(P<0.05)$

Abbreviations: VBM voxel-based morphometry, CSVD cerebral small vessel disease, IQR interquartile range, DM diabetes mellitus, WMH white matter

hyperintensity, EPVSs enlarged perivascular spaces, PSQI Pittsburgh Sleep Quality Index, BMI body mass index, AHI apnea- hypopnea index, PLMI periodic limb movements index, SDNN the standard deviation of normal-to-normal intervals, RMSSD the root mean square of successive differences in RR intervals, PNN50 the percentage of normal R-R intervals that differ by $50 \mathrm{~ms}$, LF low-frequency power, $H F$ high-frequency power

${ }^{a}$ Extensive WMH: deep WMH Fazekas 2-3(confluent or early confluent) and/or periventricular WMH Fazekas 3(extending into the deep white matter)

${ }^{\mathrm{b}}$ Moderate to extensive EPVSs in basal ganglia: $10-25$ or $>25$ EPVSs in basal ganglia

preferential atrophy of the right hemispheres in patients with arteriosclerotic CSVD. Right hemispheres have been previously reported to have a dominance of parasympathetic effects [24, 27]. In addition, the current study also suggested significant differences in PNN50, a reflection of parasympathetic function, between patients with arteriosclerotic CSVD and controls included in the VBM analysis. As a result, we posit that pathological changes in the right hemisphere may be associated with the up-regulation of sympathetic tone [27]. Actually, the underlying effects of the aforementioned brain regions on autonomic modulation are complex [14], and the current study failed to detect a loss of gray matter in other vital autonomic regions, such as the insula, hypothalamus and the anterior cingulate cortex. Accordingly, the results from our study should be interpreted with caution, and further studies with a large sample size are still necessary to provide additional evidence. 
Table 4 Regions showing differences in grey matter volume between two groups

\begin{tabular}{|c|c|c|c|c|c|c|}
\hline \multirow[t]{2}{*}{ Regions $^{*}$} & \multicolumn{2}{|c|}{ Gray matter volume, $\mathrm{mm}^{3}$, median (IQR) } & \multicolumn{3}{|c|}{ MNI Coordinates } & \multirow{2}{*}{$\begin{array}{l}\text { t- } \\
\text { value }\end{array}$} \\
\hline & CSVD group $(n=24)$ & Control group $(n=21)$ & $\bar{x}$ & $y$ & $z$ & \\
\hline Right cerebellum & $1.067(0.958,1.129)$ & $1.153(1.029,1.240)$ & 17 & -57 & -50 & 8.912 \\
\hline Left cerebellum & $1.136(1.050,1.309)$ & $1.270(1.197,1.458)$ & -14 & -63 & -51 & 8.169 \\
\hline Right inferior frontal gyrus & $2.508(2.228,2.782)$ & $2.497(2.373,2.658)$ & 33 & 29 & -6 & 7.405 \\
\hline Right thalamus & $4.117(3.096,4.813)$ & $4.861(4.407,5.354)$ & 17 & -18 & 15 & 6.500 \\
\hline Left anterior cingulate cortex. & $4.119(3.637,4.714)$ & $4.643(4.241,4.908)$ & -6 & 6 & 35 & 6.492 \\
\hline Right medial cingulate cortex & $3.858(3.440,4.267)$ & $3.858(3.758,4.142)$ & 9 & -9 & 39 & 6.155 \\
\hline Right temporal pole & $1.394(1.219,1.467)$ & $1.541(1.395,1.662)$ & 36 & 3 & -21 & 6.165 \\
\hline Right superior frontal gyrus & $10.320(9.276,10.893)$ & $9.420(9.075,9.732)$ & 24 & 45 & 23 & 5.781 \\
\hline Right lingual gyrus & $5.929(5.370,6.444)$ & $6.245(5.814,6.397)$ & 14 & -83 & -6 & 5.594 \\
\hline Left superior temporal gyrus & $5.190(4.761,5.599)$ & $5.046(4.651,5.551)$ & -45 & -24 & 5 & 5.429 \\
\hline
\end{tabular}

*Locations of maximum effect $(\mathrm{t}>4.8300 ; P<0.00001)$ was shown. Regions were automatically labeled using the AnatomyToolbox atlas. $\mathrm{x}, \mathrm{y}$, and $\mathrm{z}=\mathrm{MNI}$ coordinates in the left-right, anterior-posterior, and inferior-superior dimensions, respectively

Abbreviations: MNI Montreal Neurological Institute

Due to the design of this study, we have difficulty distinguishing whether the relationships between HRV and arteriosclerotic CSVD are bi-directional or not. Currently, the fluctuations in heart rate induced by elevated sympathetic tone are presumed to increase mechanical shear force on the vessel walls and lead to endothelial injury and arteriosclerosis [28-30]. The ensuing impairment in cerebral autoregulation and the loss of bloodbrain barrier integrity likely contribute to the development of arteriosclerotic CSVD [2, 31, 32]. Moreover, brain regions that are already damaged due to vascular deficits are likely to be nonfunctional and therefore have reduced oxygen and perfusion demands, which may accelerate the original ischemic insults and subsequent regional atrophy [33, 34]. Conversely, CSVD-related lesions may occur in fibers of the central autonomic network or contribute to the gray matter loss in brain regions associated with autonomic regulation, which may lead to a secondary imbalance between sympathetic and parasympathetic output. Studies using multimodal neuroimaging examinations are required to further verify the mechanisms described above.

As shown in our study, patients with arteriosclerotic CSVD presented greater fluctuations in nocturnal $\mathrm{SaO}_{2}$ than the control group. Due to the significant changes in $P$ values after including AHI, ODI and the interaction of

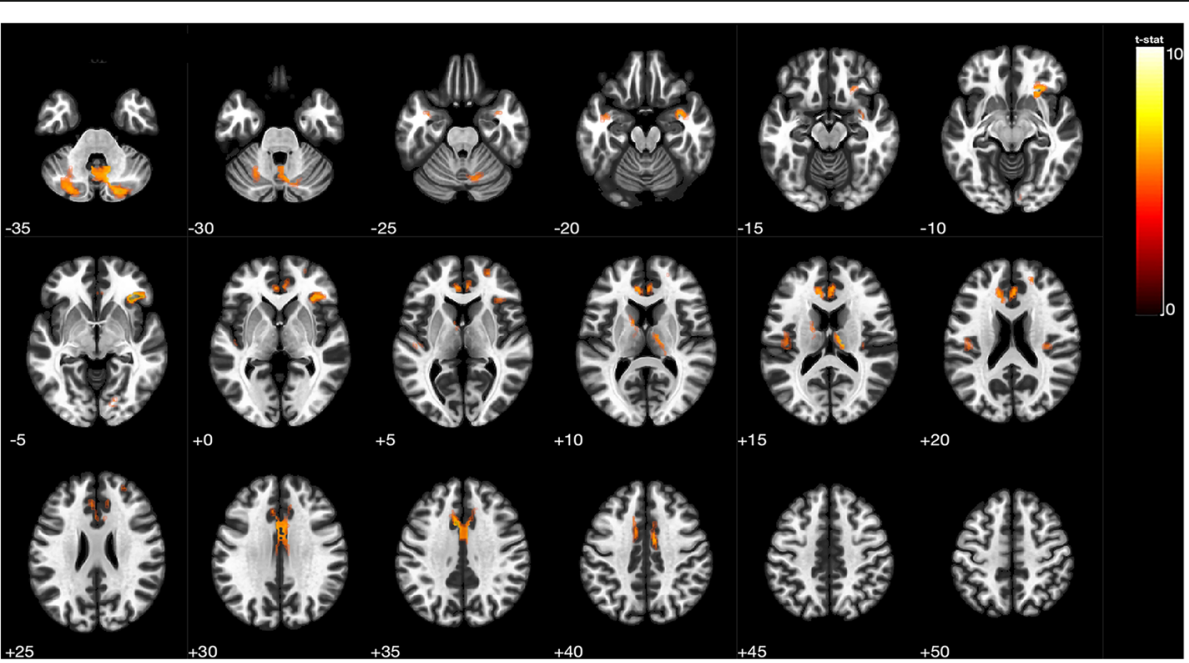

Fig. 1 Comparison of gray matter volume between CSVD patients and control participants. The numbers represents the z-axis coordinates of the corresponding plane. Red to yellow represents regions of the brain that have more significant gray matter atrophy in CSVD patients than controls at a threshold of $p<0.0001$, after adjusting for age, sex and AHI. These brain regions include the bilateral cerebellum, right superior frontal gyrus, right inferior frontal gyrus, right thalamus, right temporal pole, right superior temporal gyrus, right lingual gyrus, right medial cingulate cortex and left anterior cingulate cortex 
both parameters as covariates, a plausible hypothesis is that SDB may be involved in the relationship between HRV and arteriosclerotic CSVD. The precise role is still undetermined. According to recent studies, sympathetic overactivity assessed by monitoring HRV was associated with arteriosclerotic CSVD in patients with obstructive sleep apnea [11, 21]. Furthermore, some scholars have reported an inverse relationship between ODI and the thickness of specific cortical autonomic regions among patients with obstructive sleep apnea, suggesting that intermittent hypoxia and reoxygenation might promote cell apoptosis in some vulnerable regions of the CAN and affect the sympatho-vagal balance [35]. It is noteworthy that although significant differences existed after correcting for SDB, whether CSVD or SDB exerts more effects on HRV still needs to be further investigated.

Our study has certain limitations. The major issue is the unavoidable risk of bias due to the small and unequal sample size of the two groups, which makes multivariate modelling challenging. Moreover, the small sample sizes prevent us from further confirming the associations between HRV and arteriosclerotic CSVD using stratified analyses. Second, patients with arteriosclerotic CSVD and controls were not well gendermatched. Men are previously reported to be at higher risk of stroke and cerebral small vessel disease than women $[36,37]$, thus the difference in gender in the present study inevitably existed. Given the effect of sex on HRV [38], we have included sex as a covariate in the statistical model, accordingly we consider that the current results could be of some value. Third, heart rate has been reported to differ widely between sleep states, which exhibit a high degree of variability during REM sleep and reach its minimum during NREM sleep [39]. The current study suggested that patients with arteriosclerotic CSVD showed shorter duration of REM sleep and higher AHI throughout the whole night of sleep. Thus we speculate that sleep architecture weights less on the change of nocturnal HRV than the severity of SDB. However, we could not analyze HRV data in different sleep phases and take sleep architecture into account in the multivariable analysis due to the small sample size. Forth, although the current study has suggested a potential association between HRV and CAN among patients with arteriosclerotic CSVD, carotid atherosclerosis is considered another vital cause of the autonomic imbalance [16]. Nevertheless, we excluded patients with carotid artery stenosis $>50 \%$ and performed multivariate logistic regression analyses to adjust for traditional cerebrovascular risk factors with a $P$ value $<0.05$ in the group comparisons. What's more, a "first night effect" might exist among all participants $[40,41]$, which might reduce the significance of the difference in HRV between the two groups. Further studies are needed to verify the results.

\section{Conclusions}

In conclusion, the significantly decreased nocturnal HRV is associated with arteriosclerotic CSVD, independent of traditional cerebrovascular risk factors and SDB, suggesting sympathetic overactivity. The structural atrophy of some brain regions associated with cardiac autonomic regulation provides insights into the potential relationship. Prospective studies with larger sample sizes are required to further corroborate the conclusions.

\begin{abstract}
Abbreviations
CSVD: Cerebral small vessel disease; WMH: White matter hyperintensity: EPVSs: Enlarged perivascular spaces; CMBs: Cerebral microbleeds; HRV: Heart rate variability; ANS: autonomic nervous system; SDB: Sleep disordered breathing; CAN: Central autonomic network; MRI: Magnetic resonance imaging; CAA: Cerebral amyloid angiopathy; DM: Diabetes mellitus; BMI: Body-mass index; PSG: Polysomnography; PSQI: Pittsburgh Sleep Quality Index; NREM: Non-rapid eye movement; REM: Rapid eye movement; AASM: American Academy of Sleep Medicine; AHI: Apnea- hypopnea index; $\mathrm{SaO}_{2}$ : Oxygen saturation; ODI: Oxygen desaturation index; ST90\%: Time with $\mathrm{SaO}_{2}<90 \%$; CT90\%: Percentage of cumulative time with $\mathrm{SaO}_{2}<90 \%$; PLMI: Periodic limb movements index; ECG: Electrocardiogram;

SDNN: Standard deviation of normal-to-normal intervals; RMSSD: Root mean square of successive differences in R-R intervals; PNN50: Percentage of normal R-R intervals that differ by $50 \mathrm{~ms}$; LF: Low-frequency power; HF: Highfrequency power; 3D-BRAVO: Three-dimensional brain volume; VBM: Voxelbased morphometry; SD: Standard deviation; OR: Odds ratio; Cl: Confidence interval; GLM: Generalized linear models; SAHS: Sleep apnea-hypopnea syndrome
\end{abstract}

\section{Acknowledgements}

Not applicable.

\section{Authors' contributions}

All authors contributed to the study conception and design. Research design and participant enrollment was performed by Miaoyi Zhang and Jianhui Fu. Polysomnography records were analyzed by Huan Yu. Weijun Tang was responsible for the collection of neuroimaging data and the analysis of gray matter volume. Statistical analyses were verified by Ding Ding and Miaoyi Zhang. Material preparation and clinical data collection were performed by Jie Tang, Na Liu, Yang Xue, Xue Ren and Langfeng Shi. The first draft of the manuscript was written by Miaoyi Zhang. Writing, review and editing were performed by Jianhui Fu, and all authors commented on previous versions of the manuscript. All authors read and approved the final manuscript.

\section{Funding}

This work has been funded with grants from the National Key Research and Development Programs (2016YFC1300603; 2016YFC1301603), the Science and Technology Commission of Shanghai Municipality (17140900603, 18411962100) and the National Natural Science Foundation of China (81671151; 81901179).

\section{Availability of data and materials}

The datasets used and/or analysed during the current study are available from the corresponding author on reasonable request.

\section{Declarations}

Ethics approval and consent to participate

This study conformed with World Medical Association Declaration of Helsinki and was approved by the Huashan Hospital Research Ethics Committee (Project-ID: KY2018-224). Written informed consents for participation were obtained from all participants and their relatives.

Consent for publication

Written informed consents for publication were obtained from all participants and their relatives. 


\section{Competing interests}

The authors declare that they have no competing interests.

Received: 20 April 2021 Accepted: 30 August 2021 Published online: 16 September 2021

\section{References}

1. Wardlaw J, Smith C, Dichgans M. Mechanisms underlying sporadic cerebral small vessel disease: insights from neuroimaging. Lancet Neurol. 2013;12(5): 483-97. https://doi.org/10.1016/S1474-4422(13)70060-7.

2. Pantoni L. Cerebral small vessel disease: from pathogenesis and clinical characteristics to therapeutic challenges. Lancet Neurol. 2010;9(7):689-701. https://doi.org/10.1016/S1474-4422(10)70104-6.

3. Wardlaw JM, Smith EE, Biessels GJ, Cordonnier C, Fazekas F, Frayne R, et al. Neuroimaging standards for research into small vessel disease and its contribution to ageing and neurodegeneration. Lancet Neurol. 2013;12(8): 822-38. https://doi.org/10.1016/S1474-4422(13)70124-8.

4. Zeki Al Hazzouri A, Elfassy T, Carnethon MR, Lloyd-Jones DM, Yaffe K. Heart rate variability and cognitive function in middle-age adults: the coronary artery risk development in young adults. Am J Hypertens. 2018;31(1):27-34. https://doi.org/10.1093/ajh/hpx125.

5. Dekker JM, Crow RS, Folsom AR, Hannan PJ, Liao D, Swenne CA, et al. Low heart rate variability in a 2-minute rhythm strip predicts risk of coronary heart disease and mortality from several causes: the ARIC study. Atherosclerosis Risk In Communities. Circulation. 2000;102(11):1239-44. https://doi.org/10.1161/01.cir.102.11.1239.

6. Palatini P, Reboldi G, Beilin LJ, Eguchi K, Imai Y, Kario K, et al. Predictive value of night-time heart rate for cardiovascular events in hypertension. The ABP-International study. Int J Cardiol. 2013;168(2):1490-5. https://doi.org/1 0.1016/j.ijcard.2012.12.103.

7. Del Brutto $\mathrm{OH}$, Mera RM, Penaherrera E. The role of nighttime heart rate variability to detect white matter hyperintensities of presumed vascular origin in community-dwelling older adults. Int J Stroke. 2018;13(9):NP22NP3. https://doi.org/10.1177/1747493018806172.

8. Nakanishi K, Jin Z, Homma S, Elkind MSV, Rundek T, Lee SC, et al. Association between heart rate and subclinical cerebrovascular disease in the elderly. Stroke. 2018;49(2):319-24. https://doi.org/10.1161/STROKEAHA.11 7.019355 .

9. Song T-J, Park J-H, Choi KH, Chang Y, Moon J, Kim J-H, et al. Moderate-tosevere obstructive sleep apnea is associated with cerebral small vessel disease. Sleep Med. 2017;30:36-42. https://doi.org/10.1016/j.sleep.2016.03. 006.

10. Kang MK, Koo DL, Shin JH, Kwon HM, Nam H. Association between periodic limb movements during sleep and cerebral small vessel disease. Sleep Med. 2018;51:47-52. https://doi.org/10.1016/j.sleep.2018.06.018.

11. Del Brutto $\mathrm{OH}$, Mera RM, Costa AF, Castillo PR. Effect of heart rate variability on the association between the apnea-hypopnea index and cerebral small vessel disease. Stroke. 2019;50(9):2486-91. https://doi.org/10.1161/STROKEA HA.119.026095.

12. Sasai T, Matsuura M, Inoue $Y$. Change in heart rate variability precedes the occurrence of periodic leg movements during sleep: an observational study. BMC Neurol. 2013;13(1):139. https://doi.org/10.1186/1471-2377-13-139.

13. Valenza G, Sclocco R, Duggento A, Passamonti L, Napadow V, Barbieri R, et al. The central autonomic network at rest: uncovering functional MRI correlates of time-varying autonomic outflow. Neuroimage. 2019;197:38390. https://doi.org/10.1016/j.neuroimage.2019.04.075.

14. Beissner F, Meissner K, Bar KJ, Napadow V. The autonomic brain: an activation likelihood estimation meta-analysis for central processing of autonomic function. J Neurosci. 2013;33(25):10503-11. https://doi.org/10.1 523/JNEUROSCI.1103-13.2013.

15. Tang J, Shi L, Zhao Q, Zhang M, Ding D, Yu B, et al. Coexisting cortical atrophy plays a crucial role in cognitive impairment in moderate to severe cerebral small vessel disease patients. Discov Med. 2017;23(126):175-82.

16. Xiong L, Leung HW, Chen XY, Han JH, Leung WH, Soo OY, et al. Autonomic dysfunction in ischemic stroke with carotid stenosis. Acta Neurol Scand. 2012;126(2):122-8. https://doi.org/10.1111/j.1600-0404.2011.01617.x.

17. Rosendorff C, Lackland DT, Allison M, Aronow WS, Black HR, Blumenthal RS, et al. Treatment of hypertension in patients with coronary artery disease: a scientific statement from the American Heart Association, American College of Cardiology, and American Society of Hypertension. Hypertension. 2015; 65(6):1372-407. https://doi.org/10.1161/HYP.0000000000000018.
18. Fox CS, Golden SH, Anderson C, Bray GA, Burke LE, de Boer IH, et al. Update on prevention of cardiovascular disease in adults with type 2 diabetes mellitus in light of recent evidence: a scientific statement from the American Heart Association and the American Diabetes Association. Diabetes Care. 2015;38(9):1777-803. https://doi.org/10.2337/dci15-0012.

19. Berry RB, Brooks R, Gamaldo CE, et al. The AASM Manual for the Scoring of Sleep and Associated Events (Version 2.4)[M]. 2017.

20. Fyfe-Johnson AL, Muller CJ, Alonso A, Folsom AR, Gottesman RF, Rosamond $W D$, et al. Heart rate variability and incident stroke: the atherosclerosis risk in communities study. Stroke. 2016;47(6):1452-8. https://doi.org/10.1161/ STROKEAHA.116.012662.

21. Moon J, Choi KH, Park JH, Song TJ, Choi YS, Kim JH, et al. Sympathetic Overactivity Based on Heart-Rate Variability in Patients with Obstructive Sleep Apnea and Cerebral Small-Vessel Disease. J Clin Neurol. 2018;14(3):310-9.

22. Klarenbeek P, van Oostenbrugge RJ, Rouhl RP, Knottnerus IL, Staals J. Ambulatory blood pressure in patients with lacunar stroke: association with total MRI burden of cerebral small vessel disease. Stroke. 2013;44(11):2995-9. https://doi.org/10.1161/STROKEAHA.113.002545.

23. Yamaguchi $Y$, Wada M, Sato H, Nagasawa H, Koyama S, Takahashi $Y$, et al. Impact of nocturnal heart rate variability on cerebral small-vessel disease progression: a longitudinal study in community-dwelling elderly Japanese. Hypertens Res. 2015;38(8):564-9. https://doi.org/10.1038/hr.2015.38.

24. Winkelmann T, Thayer JF, Pohlack S, Nees F, Grimm O, Flor H. Structural brain correlates of heart rate variability in a healthy young adult population. Brain Struct Funct. 2017;222(2):1061-8. https://doi.org/10.1007/s00429-01 6-1185-1.

25. Wood KN, Badrov MB, Speechley MR, Shoemaker JK. Regional cerebral cortical thickness correlates with autonomic outflow. Auton Neurosci. 2017; 207:28-36. https://doi.org/10.1016/j.autneu.2017.05.012.

26. Ruiz Vargas E, Soros P, Shoemaker JK, Hachinski V. Human cerebral circuitry related to cardiac control: a neuroimaging meta-analysis. Ann Neurol. 2016; 79(5):709-16. https://doi.org/10.1002/ana.24642.

27. Wei L, Chen $H$, Wu GR. Heart rate variability associated with grey matter volumes in striatal and limbic structures of the central autonomic network. Brain Res. 2018;1681:14-20. https://doi.org/10.1016/j.brainres.2017.12.024.

28. Thorin E, Thorin-Trescases N. Vascular endothelial ageing, heartbeat after heartbeat. Cardiovasc Res. 2009;84(1):24-32. https://doi.org/10.1093/cvr/ cvp236.

29. Aeschbacher S, Schoen T, Dörig L, Kreuzmann R, Neuhauser C, SchmidtTrucksäss A, et al. Heart rate, heart rate variability and inflammatory biomarkers among young and healthy adults. Ann Med. 2016;49(1):32-41. https://doi.org/10.1080/07853890.2016.1226512.

30. Diaz KM, Veerabhadrappa P, Kashem MA, Thakkar SR, Feairheller DL, Sturgeon KM, et al. Visit-to-visit and 24-h blood pressure variability: association with endothelial and smooth muscle function in African Americans. J Hum Hypertens. 2013;27(11):671-7. https://doi.org/10.1038/ jhh.2013.33.

31. Fu JH, Lu CZ, Hong Z, Dong Q, Ding D, Wong KS. Relationship between cerebral vasomotor reactivity and white matter lesions in elderly subjects without large artery occlusive disease. J Neuroimaging. 2006;16(2):120-5. https://doi.org/10.1111/j.1552-6569.2006.00030.x.

32. Mestre H, Kostrikov S, Mehta Rl, Nedergaard M. Perivascular spaces, glymphatic dysfunction, and small vessel disease. Clin Sci (Lond). 2017; 131(17):2257-74. https://doi.org/10.1042/CS20160381.

33. Martinez Sosa S, Smith KJ. Understanding a role for hypoxia in lesion formation and location in the deep and periventricular white matter in small vessel disease and multiple sclerosis. Clin Sci (Lond). 2017;131(20): 2503-24. https://doi.org/10.1042/CS20170981.

34. Lambert C, Benjamin P, Zeestraten E, Lawrence AJ, Barrick TR, Markus HS. Longitudinal patterns of leukoaraiosis and brain atrophy in symptomatic small vessel disease. Brain. 2016;139(4):1136-51. https://doi.org/10.1093/bra in/aww009.

35. Taylor KS, Millar PJ, Murai H, Haruki N, Kimmerly DS, Bradley TD, Floras JS. Cortical autonomic network gray matter and sympathetic nerve activity in obstructive sleep apnea. Sleep. 2018;41(2):Zsx208.

36. Han F, Zhai FF, Wang Q, Zhou LX, Ni J, Yao M, et al. Prevalence and risk factors of cerebral small vessel disease in a Chinese population-based sample. J Stroke. 2018;20(2):239-46. https://doi.org/10.5853/jos.2017.02110.

37. Bushnell CD, Chaturvedi S, Gage KR, Herson PS, Hurn PD, Jiménez MC, et al. Sex differences in stroke: challenges and opportunities. J Cereb Blood Flow Metab. 2018;38(12):2179-91. https://doi.org/10.1177/0271678X18793324. 
38. Koenig J, Thayer JF. Sex differences in healthy human heart rate variability: a meta-analysis. Neurosci Biobehav Rev. 2016;64:288-310. https://doi.org/10.1 016/j.neubiorev.2016.03.007.

39. Harris CD. Neurophysiology of sleep and wakefulness. Respir Care Clin N Am. 2005;11 (4):567-86. https://doi.org/10.1016/j.rcc.2005.08.001.

40. Agnew HW Jr, Webb WB, Williams RL. The first night effect: an EEG study of sleep. Psychophysiology. 1966;2(3):263-6. https://doi.org/10.1111/j.14698986.1966.tb02650.x.

41. Tamaki M, Nittono H, Hayashi M, Hori T. Examination of the first-night effect during the sleep-onset period. Sleep. 2005;28(2):195-202. https://doi.org/1 0.1093/sleep/28.2.195.

\section{Publisher's Note}

Springer Nature remains neutral with regard to jurisdictional claims in published maps and institutional affiliations.

Ready to submit your research? Choose BMC and benefit from:

- fast, convenient online submission

- thorough peer review by experienced researchers in your field

- rapid publication on acceptance

- support for research data, including large and complex data types

- gold Open Access which fosters wider collaboration and increased citations

- maximum visibility for your research: over $100 \mathrm{M}$ website views per year

At BMC, research is always in progress.

Learn more biomedcentral.com/submissions 